\title{
Evaluación de pretratamientos químicos sobre materiales lignocelulósicos
}

\author{
Chemical petreatments evaluation on lignocellulosic materials
}

\author{
Diana Torres Jaramillo ${ }^{1} \quad$ Sonia Patricia Morales Vélez ${ }^{1} \quad$ Juan Carlos Quintero Díaz ${ }^{1 *}$
}

Recibido 23 de mayo de 2016, aceptado 21 de noviembre de 2016

Received: May 23, $2016 \quad$ Accepted: November 21, 2016

\begin{abstract}
RESUMEN
La eficiencia de la producción de etanol a partir de materiales lignocelulósicos depende en alto grado de la remoción de lignina, la que limita la hidrólisis de la celulosa y la posterior fermentación. En este trabajo se determinó el efecto de diferentes pretratamientos químicos sobre la composición química y cristalinidad en los materiales lignocelulósicos, aserrín de madera, bagazo de caña y cascarilla de arroz. Los pretratamientos evaluados se llevaron a cabo aplicando ácido sulfúrico o hidróxido de sodio o la combinación de ambos sobre el material.

Con la aplicación del pretratamiento combinado genera las mayores remociones de lignina. Para bagazo de caña se obtuvo una remoción de lignina del $80,6 \%$, mientras que para aserrín y cascarilla de arroz la remoción fue del 46,7\%. Sin embargo, se observó una pérdida apreciable de celulosa, del 30,7\% para el bagazo y $15,58 \%$ para la cascarilla. El análisis por difractometría de rayos X, reveló un incremento de la cristalinidad para el aserrín y el bagazo de caña.
\end{abstract}

Palabras clave: Remoción de lignina, bagazo de caña, cascarilla de arroz, aserrín, difractometría de rayos X.

\begin{abstract}
The efficiency in ethanol production from lignocellulosic materials depends on high grade on the degree of lignin removal. Lignin presence limits cellulose hydrolysis and fermentation. In this work, was determined the effect of different chemical pretreatments on chemical composition and crystallinity on the lignocellulosic materials, wood chips, cane bagasse, and rice husks. Pretreatments evaluated were carried out applying sulfuric acid or sodium hydroxide or their combination over each material. The combined pretreatment allowed to obtain the highest lignine removal. For cane bagasse it was obtained an $80.6 \%$ of lignine removal, while for wood chip and rice husks lignine was removed in $46.7 \%$. However one important cellulose loss of $30.7 \%$ was observed from bagasse cane and $15.8 \%$ from rice husks. DRX analysis showed an increase in the crystallinity for wood chip and cane bagasse.
\end{abstract}

Keywords: Lignine removal, cane bagasse, rice husk, woodchips, X-ray diffractometry.

\section{INTRODUCCIÓN}

El impacto asociado a las emisiones producidas por los combustibles fósiles, ha suscitado el interés por el estudio y desarrollo de las energías renovables como el uso de biomasa, en donde se destacan los desechos industriales y municipales, los residuos de cultivos agropecuarios y plantaciones con propósitos

\footnotetext{
1 Universidad de Antioquia. Departamento de Ingeniería Química. Grupo de Bioprocesos. Calle 67 № 53-108. Medellín, Colombia. E-mail: dianatorresj@gmail.com; sonia.morales@udea.edu.co; carlos.quintero@udea.edu.co

* Autor de correspondencia.
} 
energéticos. De ellos, la cascarilla de arroz, el bagazo de caña y el aserrín de madera son tres fuentes de gran importancia por su contenido de celulosa y su volumen de producción. En promedio la cantidad de residuos lignocelulósicos de cascarilla y el bagazo son respectivamente de $0,25 \mathrm{t}$ de cascarilla/t de arroz y 0,28 t de bagazo/t de caña de azúcar. Se puede estimar con la producción del año 2013, que en Colombia se produjeron en este año, cerca de $608.713 \mathrm{t}$ de cascarilla de arroz y $6.149 .068 \mathrm{t}$ de bagazo de caña [1]. Igualmente se producen cerca de $700.000 \mathrm{t}$ de residuos de la madera [2], todos ellos potencialmente útiles como materia prima para la producción de biocombustibles.

Para la transformación de la biomasa celulósica hasta azúcares fermentables y posteriormente a etanol u otros productos de fermentación, primero se debe realizar un pretratamiento del material con el fin de eliminar las fracciones hemicelulósica y lignina que estando presentes dificultan la acción de las enzimas celulasas responsables de la hidrólisis de la celulosa. La remoción de la fracción hemicelulósica, se realiza principalmente mediante tratamiento con ácido diluido que hidroliza la hemicelulosa a unidades de pentosas solubles en agua. También estas pentosas han sido usadas como materia prima en varios procesos químicos y biológicos, como en la producción de etanol y xilitol entre otros. La remoción de la lignina se facilita por un tratamiento alcalino que permite su solubilización. Las condiciones alcalinas además pueden cambiar la estructura cristalina de la celulosa e induce su hinchamiento facilitando la hidrólisis posterior de la celulosa [3]. El pretratamiento entonces, tiene como objetivo principal deslignificar e hidrolizar las hemicelulosas de los materiales lignocelulósicos facilitando el acceso de las enzimas celulasas sobre la celulosa para obtener azúcares fermentables. Durante el pretratamiento del material lignocelulósico, se incrementa el área superficial, se modifica la estructura y se reduce la cristalinidad de la celulosa. Se ha demostrado que el pretratamiento es una de las etapas más costosas de la producción de etanol a partir de materiales lignocelulósicos, representando entre el 15 y $20 \%$ del costo total [4].

Los pretratamientos ácidos se han llevado a cabo con ácido sulfúrico, acido clorhídrico o nítrico, aunque estos dos últimos se usan en menor proporción. Los pretratamientos con altas concentración de ácido
(30-70\%) a bajas temperaturas $\left(40{ }^{\circ} \mathrm{C}\right)$ generan problemas de corrosividad y es peligroso su manejo. Es por esto que el tratamiento con ácido diluido es más aplicado comúnmente (entre $120-190{ }^{\circ} \mathrm{C}$ y concentración de ácido sulfúrico de 0,1-1\%) [5-6].

El tratamiento alcalino se refiere a la aplicación de hidróxido de sodio, de calcio o de amonio para la remoción de lignina y parte de la hemicelulosa. Este procedimiento, generalmente genera un significativo incremento en la posterior sacarificación de la celulosa. El álcali más usado es el hidróxido de sodio con concentraciones entre $2-15 \%$ a temperaturas entre $70-121^{\circ} \mathrm{C}[5-6]$.

En la mayoría de trabajos encontrados donde se aplican pretratamientos químicos a materiales lignocelulósicos, se presentan los porcentajes de remoción de lignina y hemicelulosa, pero poca atención se da a la remoción de celulosa con estos pretratamientos, que representa una pérdida de materia prima para el proceso. Asimismo, información sobre remoción de lignina y hemicelulosa para cascarilla de arroz y aserrín es muy limitada. En este trabajo se propone evaluar la eficiencia de los pretratamientos ácidos y alcalinos individualmente, así como evaluar un pretratamiento combinado involucrando una etapa de ácido diluido seguida de una alcalina, con el fin de determinar la eficiencia de remoción de lignina, celulosa y hemicelulosa de materiales con distintas características estructurales como aserrín, bagazo de caña y cascarilla de arroz.

\section{MATERIALES Y MÉTODOS}

Residuos agroindustriales. Los materiales lignocelulósicos empleados en este estudio, aserrín, cascarilla de arroz y bagazo de caña fueron obtenidas de las siguientes fuentes: El aserrín fue una mezcla de residuos de madera de cedro, roble, teca y nogal obtenida del aserradero Aserríos de maderas el rayo (Medellín-Antioquia). La cascarilla se obtuvo como resultado de la trilla de arroz de grano medio, adquirida en la Trilladora Colombia (Central mayorista de Abastos, Medellín-Antioquia) y el bagazo de caña de la especie Saccharum officinarum (variedad cultivada para la producción de azúcar) fue obtenido del Trapiche J.M. Estrada (La Estrella-Antioquia).

Diseño experimental. Se propuso un modelo unifactorial por bloques aleatorizados completos 
con tres niveles en el factor y tres bloques. Para cada material o bloque, se evaluó el factor pretratamiento a tres niveles (T. ácido diluido, T. alcalino y T. combinado). Todos los ensayos fueron realizados por triplicado para un total de 27 experimentos. Cada material lignocelulósico se ajustó a un tamaño de partícula inferior a $3 \mathrm{~mm}$. El tratamiento con ácido se llevó a cabo en Erlenmeyer de $250 \mathrm{~mL}$, mezclando el material seco con una solución de ácido sulfúrico diluido al $10 \% \mathrm{p} / \mathrm{v}$, en una relación sólido-liquido de 1:10. La temperatura se controló en $70^{\circ} \mathrm{C}$ y se mantuvo agitación permanente durante el tiempo de tratamiento de 75 minutos. Posteriormente se lavó el material sólido residual, con abundante agua hasta neutralizar el residuo y se secó a $65^{\circ} \mathrm{C}$ por 3 horas. El tratamiento alcalino se desarrolló bajo las mismas condiciones que el ácido, pero usando hidróxido de sodio al 10\% p/v. El tratamiento combinado consistió en aplicar el tratamiento ácido y posterior a un lavado con agua se aplicó el pretratamiento alcalino con las condiciones descritas anteriormente. La efectividad de cada pretratamiento se determinó a partir del porcentaje de remoción de cada uno de los componentes de la matriz lignocelulósica (extraíbles, hemicelulosas, celulosa, lignina y cenizas) usando los resultados de la caracterización de los materiales antes y después de cada pretratamiento. El porcentaje de material eliminado $R_{i}$, en cada pretratamiento se calculó mediante la ecuación (1):

$$
R_{i}=100 *\left(M_{0 i}-M_{f i}\right) / M_{0 i}
$$

En donde:

$M_{o i}=$ Masa $(\mathrm{g})$ del componente $\mathrm{i}$ antes del pretratamiento.

$M_{f i}=$ Masa $(\mathrm{g})$ del componente i después del pretratamiento.

\section{Métodos analíticos}

Determinación de área específica. Se consideró una geometría cilíndrica para las partículas y se tomó un factor de forma de uno para todos los materiales, asumiendo iguales las dimensiones de longitud y diámetro. Las muestras de material que pasaron a través de la malla de $3 \mathrm{~mm}$ fueron tamizadas en una serie de tamices de $3,0 \mathrm{~mm} ; 2,0 \mathrm{~mm}, 1,6 \mathrm{~mm}$, $1,25 \mathrm{~mm}, 1,0 \mathrm{~mm}, 0,315 \mathrm{~mm}$ y $0,090 \mathrm{~mm}$.
Se calculó el diámetro promedio de partícula de la masa del material $D_{P}(\mathrm{~m})$ usando la ecuación (2):

$$
D_{P}=\sum D_{i}^{*} X_{i}
$$

En donde:

$D_{i}=$ Diámetro de las partículas en la i-ésima malla (m). $X_{i}=$ Fracción másica en la i-ésima malla $(\mathrm{kg} / \mathrm{kg})$.

El área específica de partículas $A_{p}\left(\mathrm{~m}^{2} / \mathrm{kg}\right)$ para los tres materiales, se determinó aplicando el método diferencial sobre el análisis granulométrico por tamizado empleando la ecuación (3).

$$
A_{P}=\frac{6}{\Phi_{S} \rho_{P}} \sum \frac{X_{i}}{D_{i}}
$$

En donde:

$\Phi_{s}=$ Factor de esfericidad de las partículas (tomado como 1).

$\rho_{P}=$ Densidad de las partículas $\left(\mathrm{kg} / \mathrm{m}^{3}\right) .(623$ $\mathrm{kg} / \mathrm{m}^{3}$ para bagazo; $652 \mathrm{~kg} / \mathrm{m}^{3}$ para cascarilla de arroz y $715 \mathrm{~kg} / \mathrm{m}^{3}$ para viruta de madera [7-8].

$D_{i}=$ Diámetro de las partículas en la i-ésima malla $(\mathrm{m})$.

Determinación de humedad. Se determinó por diferencia de peso, secando una muestra de $1 \mathrm{~g}$ previamente molida a $1 \mathrm{~mm}$ de tamaño de partícula sobre una cápsula de aluminio en una estufa a $105^{\circ} \mathrm{C}$ durante 3 horas.

Determinación de contenido de fibra. La determinación del contenido de celulosa, hemicelulosas, lignina y cenizas se realizó usando el método de Van Soest [9]. Se sometió cada muestra de material, antes y después de cada pretratamiento, a reflujo durante $1 \mathrm{~h}$, primero usando $100 \mathrm{~mL}$ de solución de detergente neutro y posteriormente $100 \mathrm{~mL}$ de solución de detergente ácido. El residuo sólido se trató con $25 \mathrm{~mL}$ de ácido sulfúrico al $72 \%$ durante 3 horas y después de un lavado con agua hasta neutralidad, el residuo se colocó en una mufla a $550{ }^{\circ} \mathrm{C}$ durante 3 horas. En cada una de estas etapas se calculó la diferencia de peso de la muestra, para determinar en su orden los porcentajes fibra detergente neutra (FDN), fibra detergente ácida (FDA), fibra detergente lignina (FDL) y cenizas; a partir de estos valores se calcularon las fracciones de componentes de la fibra usando las ecuaciones 4 a 7 . 
$\%$ Material extraíble $=100-\% F D N$

$$
\% \text { Hemicelulos } a=\% F D N-\% F D A
$$

$$
\% \text { Celulosa }=\% F D A-\% F D L
$$

$$
\% \text { Lignina }=\% F D L-\% \text { Cenizas }
$$

Determinación de cristalinidad. A cada material sin pretratar y pretratado, previamente molido, con tamaño de partícula inferior a $90 \mu \mathrm{m}$, se realizó un análisis de difractometría de rayos $\mathrm{X}$, en un difractómetro para polvos, operando a $40 \mathrm{kV}$ y $30 \mathrm{~mA}$, provisto de una fuente de $\mathrm{Cu}$ $(\lambda=1,5418 \AA)$. Las muestras se analizaron en un rango angular de $3-60^{\circ}(2 \theta)$ a $2^{\circ} / \mathrm{min}$. El índice de cristalinidad $(\mathrm{Cr} I)$ se determinó por medio de la relación dada por la ecuación (8), entre los picos máximos característicos de la celulosa utilizando las intensidades de difracción de la estructura cristalina (plano $2 \theta=22,7^{\circ}$ ) y de la fracción amorfa (plano $\left.2 \theta=17,0^{\circ}\right)[10]$.

$$
C r I=100 *\left(\frac{I_{002}-I_{\text {amorfa }}}{I_{002}}\right)
$$

En donde:

$I_{002}=$ Intensidad del pico en el máximo de $2 \theta$ entre $22^{\circ}$ y $23^{\circ}$ para celulosa del tipo I y entre $20-22^{\circ}$ para celulosa tipo II.
$I_{\text {amorfa }}=$ Intensidad del pico en el mínimo de $2 \theta$ entre $15^{\circ}$ y $17^{\circ}$ para celulosa del tipo I entre $12-13^{\circ}$ para celulosa tipo II.

\section{RESULTADOS}

Caracterización del material. Los valores de diámetro de partícula y área específica para los tres materiales se presentan en la Tabla 1. El área específica es una de las principales características que permite una mayor eficiencia en los pretratamientos y en la sacarificación. El bagazo de caña y el aserrín, presentan las mayores áreas específicas que pueden permitir mejores condiciones para la remoción de lignina y hemicelulosa. El área específica de la cascarilla de arroz es significativamente menor, en un $40 \%$, que la del aserrín y el bagazo de caña.

Tabla 1. Características morfológicas de los materiales lignocelulósicos.

\begin{tabular}{|c|c|c|c|}
\hline Características & Aserrín & Bagazo & Cascarilla \\
\hline Diámetro promedio $(\mathrm{m})$ & $8,5 \mathrm{E}-04$ & $6,9 \mathrm{E}-04$ & $1,3 \mathrm{E}-03$ \\
\hline Área espeć́fica $\left(\mathrm{m}^{2} / \mathrm{kg}\right)$ & 20,1 & 19,6 & 12,0 \\
\hline
\end{tabular}

La caracterización química por el método de Van Soest realizada antes y después de los pretratamientos aplicados se presenta en la Tabla 2. Se observa que el porcentaje de celulosa para los tres materiales es muy similar y se encuentra entre el 32 y $36 \%$. Después de cada pretratamiento se encuentra un incremento en el porcentaje de celulosa en el material, lo que se

Tabla 2. Resultados de composición química de aserrín, bagazo de caña y cascarilla de arroz antes y después de cada pretratamiento.

\begin{tabular}{|l|l|r|r|r|r|r|}
\cline { 3 - 7 } \multicolumn{2}{c|}{} & \multicolumn{5}{c|}{ Porcentajes de cada componente (\% en peso seco) } \\
\hline \multicolumn{1}{|c|}{ Material } & Pretratamiento & Celulosa & Hemicelulosa & Lignina & Extraíbles & Cenizas \\
\hline \multirow{4}{*}{ Aserrín } & Ninguno & $36,6 \pm 0,9$ & $7,0 \pm 0,7$ & $38,8 \pm 0,5$ & $17,5 \pm 0,2$ & $0,4 \pm 0$ \\
\cline { 2 - 7 } & Ácido & $42,8 \pm 0,5$ & $6,3 \pm 0,4$ & $37,5 \pm 0,9$ & $12,9 \pm 0,4$ & $0,4 \pm 0$ \\
\cline { 2 - 7 } & Básico & $48,7 \pm 0,3$ & $7,3 \pm 0,8$ & $33,8 \pm 0,7$ & $9,8 \pm 0,5$ & $0,4 \pm 0$ \\
\cline { 2 - 7 } & Combinado & $57,6 \pm 0,7$ & $4,7 \pm 0,7$ & $27,6 \pm 0,4$ & $9,7 \pm 0,3$ & $0,3 \pm 0$ \\
\hline \multirow{4}{*}{ Bagazo de caña } & Ninguno & $32,7 \pm 0,7$ & $21,3 \pm 1,3$ & $15,3 \pm 0,5$ & $29,4 \pm 0,7$ & $1,3 \pm 0,1$ \\
\cline { 2 - 7 } & Ácido & $51,7 \pm 0,6$ & $14,9 \pm 0,7$ & $16,1 \pm 0,3$ & $16,2 \pm 0,4$ & $1,1 \pm 0$ \\
\cline { 2 - 7 } & Básico & $73,0 \pm 0,5$ & $10,1 \pm 0,3$ & $7,4 \pm 0,3$ & $8,4 \pm 0,2$ & $1,1 \pm 0$ \\
\cline { 2 - 7 } & Combinado & $75,1 \pm 0,3$ & $6,4 \pm 0,3$ & $7,9 \pm 0,1$ & $9,4 \pm 0,3$ & $1,2 \pm 0$ \\
\hline \multirow{4}{*}{ Cascarilla de arroz } & Ninguno & $34,4 \pm 0,1$ & $9,5 \pm 0,2$ & $22,1 \pm 0,4$ & $16,2 \pm 0,6$ & $17,7 \pm 0,1$ \\
\cline { 2 - 7 } & Ácido & $43,5 \pm 1,1$ & $7,9 \pm 0,9$ & $19,6 \pm 1,6$ & $10,8 \pm 0,9$ & $17,9 \pm 0,4$ \\
\cline { 2 - 7 } & Básico & $44,2 \pm 2,4$ & $6,8 \pm 0,8$ & $16,2 \pm 2,0$ & $10,2 \pm 0,5$ & $22,5 \pm 0,3$ \\
\cline { 2 - 7 } & Combinado & $58,4 \pm 1,0$ & $7,0 \pm 0,5$ & $12,0 \pm 0,8$ & $7,3 \pm 0,2$ & $15,2 \pm 0,3$ \\
\hline
\end{tabular}


debe a la reducción de las fracciones hemicelulósica y lignina principalmente. El mayor porcentaje de celulosa y el menor contenido de hemicelulosa y lignina, se obtiene en los materiales que fueron pretratados con la combinación de ácido y álcali. Adicionalmente se observa que la cascarilla de arroz muestra un elevado contenido de cenizas con respecto a los otros dos materiales.

Efecto de los pretratamientos. En la Figura 1 se presentan los porcentajes de remoción de los componentes principales celulosa, hemicelulosa y la lignina del aserrín, bagazo de caña y cascarilla de arroz. Estos valores fueron calculados mediante un balance de materia de cada componente realizado en cada pretratamiento.

Se encontró que los pretratamientos alcalino y combinado son significativamente superiores para la remoción de los componentes principales de los materiales. Entre los materiales evaluados, el aserrín fue el que exhibió una mayor recalcitrancia, alcanzando un máximo de remoción de 46,7\% para hemicelulosa y también para lignina con el tratamiento combinado. Aquí mismo se observa que aunque este tratamiento es bastante exigente, no hubo pérdida de celulosa, por lo que este pretratamiento en dos etapas es el más adecuado para el aserrín. El bagazo de caña por el contrario fue el material más susceptible a los pretratamientos obteniéndose una remoción de 73,9\% para la fracción hemicelulosa, del $80,6 \%$ para la lignina y del $9,9 \%$ para la celulosa, con el tratamiento alcalino. Con el tratamiento combinado, aunque se logra una mayor remoción de la fracción hemicelulósica hasta del $80 \%$, también se observa una elevada pérdida de la fracción celulósica hasta de un 30\%, lo que hace poco adecuada la combinación de los dos pretratamientos.

De otro lado, con el aserrín se encontró un comportamiento aditivo en la remoción de hemicelulosa y lignina cuando se aplica el pretratamiento combinado con relación a los pretratamientos por separado. Mientras con el combinado se eliminó un 46,7\% de lignina, en el pretratamiento ácido se eliminó el $17 \%$ y en el alcalino el $32 \%$. Este fenómeno no se observó con los otros materiales.

En la Figura 2 se observan los resultados del análisis por DRX de los 3 sustratos lignocelulósicos antes y después del pretratamiento alcalino. En los difractogramas se observa un pico característico de la fase amorfa en $17^{\circ}$ y un pico característico de la fase cristalina en $23^{\circ}$. La intensidad en señal de las fases cristalina y amorfa de la celulosa para bagazo de caña y cascarilla de arroz son mayores cuando el material ha sido pretratado, indicando un

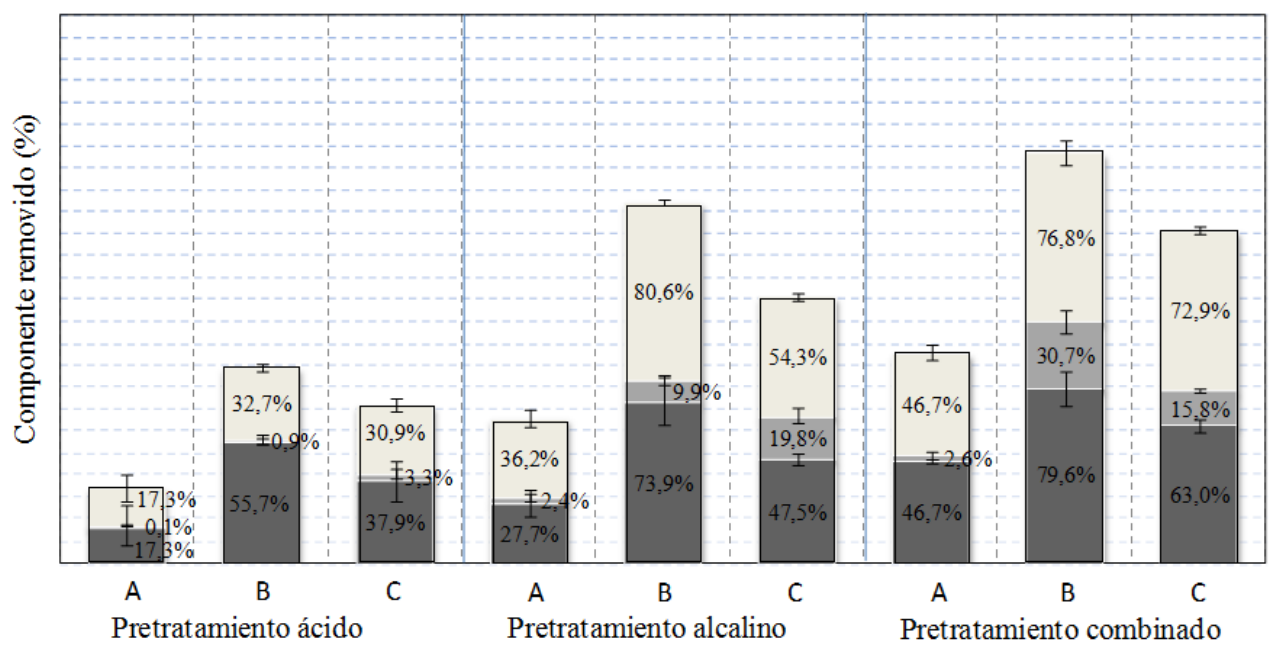

Figura 1. Porcentaje de remoción (peso seco) de los componentes de los tres materiales lignocelulósicos: (A) aserrín, (B) bagazo de caña y (C) cascarilla de arroz, empleando tres pretratamientos químicos.

$\square$ Hemicelulosa $\square$ Celulosa $\square$ Lignina. 
enriquecimiento en este tipo de material. Mientras que el aserrín pretratado presenta un difractograma con una señal menor que sin pretratamiento.

En la Tabla 3 se muestra el efecto del pretratamiento alcalino de cada material sobre la cristalinidad. Estos valores fueron calculados con la ecuación 8, a partir de la Figura 2. Posterior al pretratamiento con hidróxido de sodio, se encontró un aumento leve en la cristalinidad para el aserrín y mucho más significativo para bagazo de caña, mientras que se observó una reducción con cascarilla de arroz.

\section{DISCUSIÓN}

Caracterización. El aumento en los porcentajes de celulosa después de la aplicación de los pretratamientos ácido, alcalino y combinado para los tres materiales, es consecuencia de la eliminación de hemicelulosas y lignina del material lignocelulósico, lo que deja mayor proporción de celulosa en el residuo (Tabla 2). El mayor incremento en la proporción de celulosa fue alcanzada con el bagazo de caña en el pretratamiento combinado que pasó del $32 \%$ en el material sin pretratar al $75,1 \%$ en el pretratado. Un resultado muy similar del $73 \%$ se obtuvo con el pretratamiento alcalino. Lo anterior correlaciona muy bien con la mayor área específica de este material. El aserrín con un área específica similar a la del bagazo de caña; sin embargo, no mostró un incremento similar en el porcentaje de celulosa (21\%), indicando una baja deslignificación.

Lo anterior se puede explicar porque el aserrín presenta una estructura altamente lignificada con un $38 \%$ de lignina (Tabla 2) y además, por contener una proporción de cedro (planta gimnosperma), su lignina posee una alta relación de las unidades guayacilo/
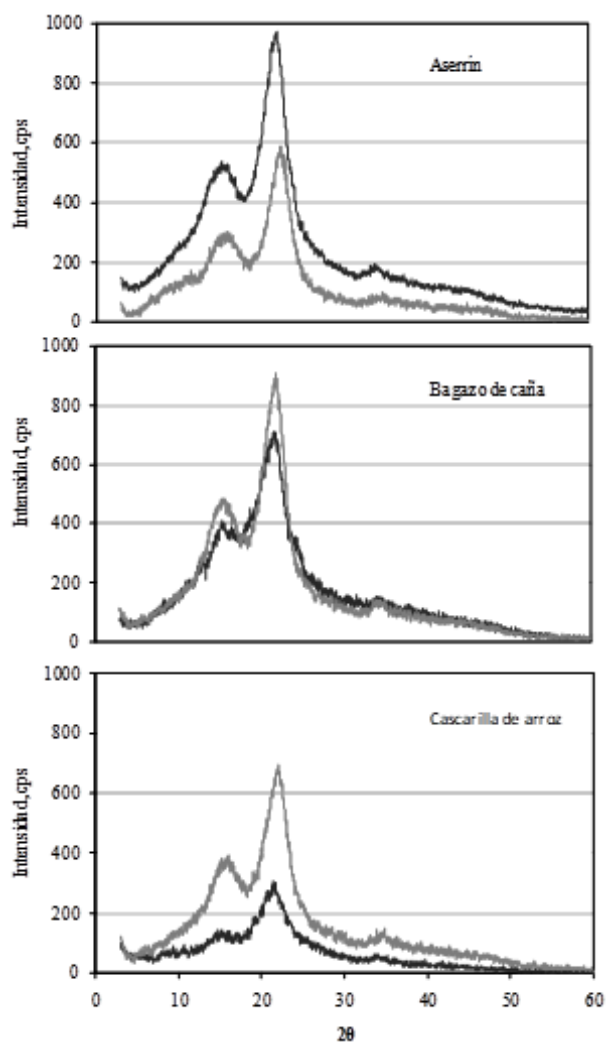

Figura 2. Difractogramas de los materiales lignocelulósicos con y sin pretratamiento alcalino. Línea oscura: material sin pretratamiento; línea clara: material pretratado.

siringilo (G/S) que dificulta su remoción [11]. Por otro lado, la lignina del bagazo de caña contiene además de unidades $\mathrm{G}$ y $\mathrm{S}$, residuos derivados del alcohol trans-p-cumarílico permitiendo un fácil proceso de deslignificación [12]. La cascarilla de arroz, presentó un incrementó de $24 \%$ en el

Tabla 3. Intensidad de las señales principales por DRX para los 3 materiales lignocelulósicos y efecto del pretratamiento alcalino sobre el índice de cristalinidad del material.

\begin{tabular}{|c|c|c|c|c|c|c|}
\cline { 2 - 7 } \multicolumn{1}{c|}{} & \multicolumn{2}{c|}{ Aserrín } & \multicolumn{2}{c|}{ Bagazo de caña } & \multicolumn{2}{c|}{ Cascarilla de arroz } \\
\hline $\begin{array}{c}\text { Intensidad } \\
\text { del pico }(\mathbf{c p s})\end{array}$ & $\begin{array}{c}\text { Sin } \\
\text { pretratar }\end{array}$ & $\begin{array}{c}\text { Pretratado con } \\
\text { NaOH 10\% }\end{array}$ & $\begin{array}{c}\text { Sin } \\
\text { pretratar }\end{array}$ & $\begin{array}{c}\text { Pretratado con } \\
\text { NaOH 10\% }\end{array}$ & $\begin{array}{c}\text { Sin } \\
\text { pretratar }\end{array}$ & $\begin{array}{c}\text { Pretratado con } \\
\text { NaOH 10\% }\end{array}$ \\
\hline $2 \theta=14,5^{\circ}$ & 958,4 & 561,5 & 705,8 & 909,8 & 305,8 & 694,9 \\
\hline $2 \theta=22,7^{\circ}$ & 530,2 & 296,5 & 390,6 & 296,5 & 144,3 & 376,5 \\
\hline Índice de cristalinidad & 0,44 & 0,47 & 0,44 & 0,67 & 0,52 & 0,45 \\
\hline
\end{tabular}


contenido de celulosa. Este material además de contener una área específica significativamente menor que el bagazo y el aserrín, que minimiza el contacto entre el álcali y la lignina, también posee una mayor proporción de unidades guayacilo con la consecuente mayor recalcitrancia de su lignina [13]. Esto demuestra que la efectividad de los pretratamientos, depende principalmente de la complejidad química y estructural de los polímeros presentes en la lignocelulosa que varían con el origen y tipo de material.

Efecto de los pretratamientos. El pretratamiento ácido en general, es más selectivo para remover la hemicelulosa que la lignina [6], lo que se confirmó para el bagazo y la cascarilla, mientras que con el aserrín la diferencia no fue significativa. Con pretratamiento ácido diluido se ha reportado la extracción de hemicelulosas entre $79,6 \%$ y $96 \%$ en aserrín (coníferas) [14, 15], 80 y $90 \%$ en bagazo de caña $[16,17]$ y 82 y $100 \%$ en cascarilla de arroz $[18$, 19] todos estos valores superiores a los encontrados en este trabajo, esto se debe a que los tratamientos se han hecho a temperaturas entre 120 y $190{ }^{\circ} \mathrm{C}$, mientras que en este trabajo se realizaron a $70^{\circ} \mathrm{C}$, por tanto, se puede concluir que mayor remoción de hemicelulosa se obtiene con concentraciones de ácido entre 0,1 y $1 \%$ y altas temperaturas, que concentraciones de ácido del $10 \%$ y temperaturas de $70{ }^{\circ} \mathrm{C}$; sin embargo, estos pretratamientos, tienden a formar sustancias inhibidoras del proceso fermentativo, son más costosos y requieren equipamiento diseñado con materiales especiales para evitar procesos de corrosión [28].

El pretratamiento alcalino, que facilita la solubilización de la lignina, tampoco fue eficiente para el aserrín, se requiere del tratamiento combinado para obtener remociones importantes de lignina y hemicelulosa. La presencia de lignina en las plantas tiene como objetivo brindar soporte, impermeabilidad y resistencia al ataque químico y microbiano. Estas propiedades varían con el tipo de precursores de la lignina (p-coumaril alcohol, coniferil-alcohol o sinapil alcohol que, a su vez, interfieren en su solubilidad en medio neutro, ácido o alcalino (4). Como se observa en la Tabla 2, el aserrín contiene una mayor cantidad de lignina $(38,8 \%)$, que hace que el material se vea afectado en menor grado por cualquiera de los tratamientos químicos (Figura 1), en comparación con el bagazo de caña, cuya proporción de lignina es baja $(15,3 \%)$ y con la cascarilla de arroz $(22,1 \%)$. Debido a que el aserrín es una mezcla de varias gimnospermas y estas a su vez están formadas mayoritariamente por unidades de guayacilo (alcohol coniferílico) que incrementan la formación de enlaces tipo éter y carbono-carbono, este tipo de maderas es más difícil de deslignificar. El bagazo de caña y la cascarilla de arroz presentan dos grupos metoxilo en las posiciones 3 y 5 del anillo aromático características de las angiospermas y cuyas unidades de siringilo presentes en la lignina presentan menor cantidad de enlaces tipo éter y carbono-carbono [20].

Los resultados de la Figura 1 también muestran que la celulosa es resistente al ataque con ácido diluido; sin embargo, con pretratamiento alcalino, para el bagazo y la cascarilla, se observó una importante remoción de celulosa, que se incrementó en el pretratamiento combinado. Se concluye, por tanto, que este último pretratamiento es el más eficiente para el aserrín, pues es donde se obtienen los mayores porcentajes de remoción de lignina y hemicelulosa y muy baja pérdida de celulosa, tan solo $2,6 \%$. Mientras que para el bagazo de caña el tratamiento más adecuado es el alcalino, por su alta remoción de lignina y hemicelulosa y su relativamente baja pérdida de celulosa, cerca del $10 \%$. En la cascarilla de arroz se observó una elevada pérdida de celulosa en los pretratamientos alcalino y combinado, y una baja remoción de lignina y hemicelulosa en el ácido. Por tanto, para este material no se considera adecuado ninguno de estos procedimientos. Estos resultados permiten concluir que no existe una única tecnología de pretratamiento aplicable diferentes tipos de biomasas.

La efectividad del pretratamiento con hidróxido de sodio aplicado en este estudio, también ha sido citada por varios autores, demostrando que puede obtenerse importantes porcentajes de deslignificación y mayor conversión enzimática de la celulosa. Con este pretratamiento se han reportado entre hasta $55 \%$ de remoción de lignina en aserrín (coníferas) [2122], valores superiores al $41 \%$ en bagazo de caña [23-24] y tan solo del $28 \%$ en cascarilla de arroz [20]. Los resultados obtenidos en este trabajo están dentro de estos rangos salvo para la cascarilla donde se obtuvo un $54 \%$ de delignificación. Debido a la elevada pérdida de celulosa, es importante considerar otra estrategia de pretratamiento para la cascarilla 
de arroz, de igual forma se podría proponer para el aserrín ya que aunque el tratamiento combinado fue el mejor, este resulta costoso. En los últimos años se ha venido desarrollando la aplicación de pretratamientos biológicos con hongos de la pudrición blanca de la madera, por su alta selectividad hacia la remoción de la lignina, facilitando un posterior tratamiento ácido para eliminar la hemicelulosa, dejando intacta la celulosa [25].

Se realizó un estudio para analizar el cambio de la estructura de los tres materiales sometidos al pretratamiento alcalino, por difractometría de rayos $\mathrm{X}$ (DRX). La celulosa es un complejo polimérico con áreas cristalinas y amorfas y la DRX permite cuantificar el grado de cristalinidad del material, que es de gran importancia para la etapa de sacarificación de la celulosa, ya que esta es más eficiente en presencia de estructuras amorfas de celulosa. Los resultados del análisis por difractometría de rayos $\mathrm{X}$ son presentados en la Figura 2.

De acuerdo con los índices de cristalinidad calculados, se observó que la cascarilla de arroz con un índice de 0,52 es la estructura con mayor proporción de regiones cristalinas en comparación con el bagazo de caña y el aserrín que presentaron un índice de 0,44 . Un mayor índice de cristalinidad también podría presentarse en un material con mayor proporción de celulosa; sin embargo, en los tres materiales el porcentaje de celulosa es similar, esto implicaría que la cristalinidad podría provenir de una mayor proporción de celulosa cristalina o del aporte a la cristalinidad de sílice presente. En el caso del aserrín, aunque de acuerdo con los índices calculados, el grado de cristalinidad de la celulosa es igual al bagazo de caña, el pretratamiento y la sacarificación posiblemente se dificultarían por su elevado contenido de lignina, concluyéndose que con las condiciones de tratamiento estudiadas en el presente trabajo, el bagazo es el material más adecuado para una posterior etapa de sacarificación.

El hidróxido de sodio se adsorbe sobre la celulosa y genera un hinchamiento de la misma, los cambios en su estructura se pueden observar por DRX. Los difractogramas del aserrín antes y después del tratamiento alcalino (ver Figura 2) no muestran cambios significativos en los patrones indicando que la estructura de la celulosa se mantuvo intacta. Esto se correlaciona bastante bien con el índice de cristalinidad (ver Tabla 3) que muestra un incremento de solo un 6,8\% en la cristalinidad de la muestra. Para el bagazo de caña y la cascarilla de arroz, los difractogramas mostraron cambios importantes, pudiéndose observar incremento en la intensidad de las fracciones cristalinas y amorfas de la celulosa después de los tratamientos alcalinos. Para el bagazo de caña la cristalinidad se incrementó en un $52 \%$, que se explica por la elevada remoción de lignina y hemicelulosa alcanzada, debido a que estos son materiales amorfos. Para la cascarilla de arroz, la cristalinidad disminuyó en un $13 \%$, lo que quiere decir que se incrementó la proporción de la fase amorfa del material. Para este material, el tratamiento alcalino removió cerca del $20 \%$ de la celulosa, lo que seguramente influyó en la pérdida de cristalinidad del material. Otros estudios realizados sobre residuos lignocelulósicos han mostrado que pretratamientos alcalinos remueven importantes cantidades de celulosa además de lignina y hemicelulosa y muestran que dependiendo del material, puede aumentar o disminuir la cristalinidad de la celulosa después del pretratamiento. Yanfeng y colaboradores (2008) (21) al tratar paja de arroz con $\mathrm{NaOH}$ al $6 \%$, encontraron una remoción de $36,8 \%$ de la hemicelulosa, $28,4 \%$ de lignina y un $16,4 \%$ de celulosa, que son resultados similares a los obtenidos en este trabajo para cascarilla de arroz. También encontraron que el pretratamiento alcalino incrementa en un $4 \%$ la cristalinidad del residuo, y esto se atribuye a una remoción preferencial de las fases amorfas de hemicelulosa y lignina, sin observarse ningún cambio en la morfología de la celulosa. Estos últimos resultados contrastan con los encontrados para cascarilla de arroz, indicando que el efecto del álcali sobre la cristalinidad del residuo puede variar con los diferentes componentes lignocelulósicos de la planta.

De otro lado, Borysiak y Doczekalska (2005) [10] encontraron una reducción del 16\% en la cristalinidad en madera de pino con tratamiento de $\mathrm{NaOH}$ al $10 \%$ e indican que se presentó esta reducción debido a una modificación polimórfica de la celulosa cristalina tipo I a celulosa cristalina tipo II, la que es más favorable energéticamente para la hidrólisis. Durante el tratamiento con soluciones de $\mathrm{NaOH}$, las moléculas de celulosa presentan un estado de hinchamiento debido a la formación de complejos álcali-celulosa $\left(\mathrm{C}_{6} \mathrm{H}_{10} \mathrm{O}_{5}-\mathrm{NaOH}-3 \mathrm{H}_{2} \mathrm{O}\right)$. Posterior al tratamiento, cuando la celulosa se lava con agua, 
esta recristaliza en una forma antiparalela para formar la celulosa tipo II [26]. Estos resultados de Botysiak y Doczekalska son concordantes con la reducción del $13 \%$ en la cristalinidad de la cascarilla de arroz encontrados en este trabajo; sin embargo, en el caso de la cascarilla no es probable que se haya presentado este cambio de estructura de la celulosa debido a que no se evidencia en el patrón de difracción de rayos $\mathrm{X}$ los picos característicos de celulosa tipo II correspondiente al plano $2 \theta=12,1^{\circ}$ y $22^{\circ}$ [27]. Esto se explica debido a que se ha observado que la transición polimórfica de la celulosa se presenta con tratamientos de $\mathrm{NaOH}$ a concentraciones superiores al 11\% [26-27] lo que hace que los resultados de Botysiak y Doczekalska sean atípicos.

Los pretratamientos químicos sobre materiales lignocelulósicos a bajas temperaturas resultan adecuados dado que así se limita la producción de sustancias inhibidoras de la fermentación, en especial con el tratamiento alcalino [29]. Pretratamientos químicos a bajas temperaturas han mostrado ser apropiados para remover la lignina y reducir la cristalinidad del material, mejorando así su posterior digestibilidad. Tratamientos con peróxido de hidrógeno y ácido acético a $80^{\circ} \mathrm{C}$ sobre madera de pino, han mostrado una alta remoción de lignina alcanzando rendimientos en la fermentación alcohólica del 84\% [28]. De la misma manera, pretratamientos con $\mathrm{KOH} \mathrm{a} 60^{\circ} \mathrm{C}$ sobre residuos de maíz, generaron conversión de celulosa cristalina en amorfa y degradación de la lignina, produciendo una alta digestibilidad anaerobia de los residuos pretratados [30].

\section{CONCLUSIONES}

Los tres materiales estudiados, cascarilla de arroz, bagazo de caña y aserrín de madera, presentan contenidos similares de celulosa en torno al 32-36\%. La cascarilla de arroz presenta altos contenidos de cenizas provenientes de su contenido de sílice. El pretratamiento ácido diluido genera una pobre remoción de lignina y hemicelulosa en todos los materiales, aunque se muestra selectivo hacia la hemicelulosa. El pretratamiento combinado, ácido diluido seguido de álcali, es bastante riguroso, pues si bien se presentan altos niveles de remoción de lignina y hemicelulosa, también se presenta una pérdida importante de celulosa en bagazo y cascarilla. Para aserrín el tratamiento combinado es el más adecuado. El bagazo de caña perdió el $80 \%$ de la lignina y cerca del $75 \%$ de la hemicelulosa en el tratamiento alcalino, con una baja remoción de celulosa, lo que hace adecuado para el pretratamiento de este material. En los tres materiales, se presentaron cambios en el contenido de fase cristalina atribuidos a la pérdida de la fase amorfa por remoción de la lignina. Este trabajo muestra como la cascarilla de arroz se presenta como un material potencial para su aprovechamiento en producción de biocombustibles por su alta remoción de lingina y baja pérdida de celulosa en el tratamiento.

\section{AGRADECIMIENTOS}

Los autores agradecen el apoyo al Comité para el Desarrollo de la Investigación CODI de la Universidad de Antioquia con el programa estrategia de sostenibilidad 2014-2015.

\section{REFERENCIAS}

[1] Ministerio de Agricultura y Desarrollo Rural. Estadísticas Agroforestales. 1987-2013. Fecha de consulta: 6 de abril de 2016. URL: http:// www.agronet.gov.co/Paginas/estadisticas.aspx

[2] C.P. Cubillos. "Disposicion de Residuos Industriales Maderables: Una Estrategia Empresarial Ambiental para Convertir Costos en Inversiones". Trabajo de Grado. 2015. Universidad Militar Nueva Granada.

[3] A.T. Hendriks and G. Zeeman. "Pretreatments to enhance the digestibility of lignocellulosic biomass". Bioresour. Technol. Vol. $100 \mathrm{~N}^{\circ} 1$, pp. 10-18. 2009.

[4] D. Humbird, R. Davis, L. Tao, C. Kinchin, D. Hsu and A. Aden. "Process Design and Economics for Biochemical Conversion of Lignocellulosic Biomass to Ethanol". National Renewable Energy Laboratory. Technical Report. 2011. Fecha de consulta: 6 de abril de 2016. URL: http://www.nrel. gov/docs/fy11osti/47764.pdf

[5] M.J. Taherzadeh and K. Karimi. "Pretreatment of lignocellulosic wastes to improve ethanol and biogas production: A review". Int. J. Mol. Sci. Vol. 9 No 9, pp. 1621-51. 2008.

[6] R.A. Silverstein, Y. Chen Y, R.R. SharmaShivappaa, M.D. Boyetteac and J. Osborneb. "A comparison of chemical pretreatment methods for improving saccharification of 
cotton stalks". Bioresour. Technol. Vol. 98 pp. 3000-11. 2007.

[7] J. Chave, D. Coomes, S. Jansen, S. Lewis, N. Swenson and A.E. Zanne. "Towards a worldwide wood economics spectrum". Ecol. Lett. Vol. $12 \mathrm{~N}^{\circ}$ 4, pp. 351-366. 2009.

[8] R. Pire y A. Pereira. "Propiedades físicas de componentes de sustratos de uso común en la horticultura del estado Lara, Venezuela. Propuesta metodológica”. Bioagro. Vol. 15 $\mathrm{N}^{\circ}$ 1, pp. 55-63. 2003.

[9] F. Segura, R. Echeverri, A.C. Patino y A. Mejía. "Descripción y discusión acerca de los metodos de analisis de fibra y del valor nutricional de forrajes y alimentos para animales." Revista VITAE. Vol. $14 \mathrm{~N}^{\mathrm{o}} 1$, pp. 72-81. 2007.

[10] S. Borysiak and B. Doczekalska. "X-ray diffraction study of pine wood treated with $\mathrm{NaOH}$ ". Fibres Text. East. Eur. Vol. $13 \mathrm{~N}^{\circ} 5$, pp. 87-89. 2005.

[11] Y.Zhao, Y. Wang, J.Y.Zhu, A. Ragauskas and Y. Deng. "Enhanced enzymatic hydrolysis of spruce by alkaline pretreatment at low temperature". Biotechnol. Bioeng. Vol. 99 $\mathrm{N}^{\circ}$ 6, pp. 1320-8. 2008.

[12] J. Sun, X. Sun, R. Sun, P. Fowler, M. Baird. "Inhomogeneities in the chemical structure of sugarcane bagasse lignin". J. Agric. Food Chem. Vol. $51 \mathrm{~N}^{\circ} 23$, pp. 6719-25. 2003.

[13] Z.A.Saipov and K.A. Abduazimov. "The lignins of ripe rice-plant stems and of rice husks". Chem. Nat. Compd. Vol. $18 \mathrm{~N}^{\circ} 6$, pp. 765-6. 1982.

[14] J. Soderstrom, L. Pilcher, M. Galbe and G. Zacchi. "Two-step steam pretreatment of softwood by dilute $\mathrm{H}_{2} \mathrm{SO}_{4}$ impregnation for ethanol production". Biomass Bioenergy. Vol. 24, pp. 475-86. 2003.

[15] E. Vieira, G.J. de Moraes, J.A. de Carvalho Jr. and J.B. de Almeida. "Optimization of acid hydrlysis from the hemicellulosic fraction of Eucalytus grandis residue using response surface methodology". Bioresour. Technol. Vol. $98 \mathrm{~N}^{\circ}$ 2, pp. 422-8. 2007.

[16] P. Das, A. Ganesh and P. Wangikar. "Influence of pretreatment for deashing of sugarcane bagasse on pyrolysis products". Biomass Bioenergy. Vol. $27 \mathrm{~N}^{\circ}$ 5, pp. 445-57. 2004.

[17] B.P. Lavarack, G.J, Griffin and D. Rodman. "The acid hydrolysis of sugarcane bagasse hemicellulose to produce xylose, arabinose, glucose and other products". Biomass Bioenergy. Vol. 23 No 5, pp. 367-80. 2002.

[18] G.L. Guo, W.H. Chen, L.C. Men and W.S. Hwang. "Characterization of dilute acid pretreatment of silver grass for ethanol production". Bioresour. Technol. Vol. 99 $\mathrm{N}^{\circ} 14$, pp. 6046-53. 2008.

[19] K. Holtman, D. Franqui, W.J. Orts, Nader and P. Robinson. "Enzymatic hydrolysis and fermentation of broken rice kernels and rice straw". Albany: Final report. United States Department of Agriculture 2008. Fecha de consulta: 6 de abril de 2016. URL: http:// www.carrb.com/08rpt/Holtman\%2008.pdf

[20] H. Yanfeng, P. Yunzhi, L. Yanping, L. Xiujin and W. Kuisheng. "Physicochemical characterization of rice straw pretreated with sodium hydroxide in the solid state for enchancing biogas production". Energy Fuels. Vol. 22, pp. 2775-2781. 2008.

[21] Y. Zhao. "Enhanced enzymatic hydrolysisof spruce by alkaline pretreatment at low temperature". Biotechnol. Bioeng. Vol. 99 $\mathrm{N}^{\circ}$ 6, pp. 1320-1328. 2008.

[22] O.J. Sanchez y C.A. Cardona. "Producción biotecnológica de alcohol carburante I: Obtención a partir de diferentes materias primas". Interciencia. Vol. $30 \mathrm{~N}^{\circ} 11$, pp. 671 678. 2005.

[23] C. Alves, A. M. de Lima, P. Maziero, R. E. de Azevedo, W. Garcia and I. Polikarpov. "Chemical and morphological characterization of sugarcane bagasse submitted to a delignification process for enhanced enzymatic digestibility". Biotechnol. Biofuel. Vol. 4, pp. 1-19. 2011.

[24] F. Ahmadi, M.J. Zamiri, M. Khorvash, E. Ziaee and I. "Pre-treatment of sugarcane bagasse with a combination of sodium hydroxide and lime for improving the ruminal degradability: optimization of process parameters using response surface methodology". J. Appl. Anim. Res., Vol. 44 $\mathrm{N}^{\circ}$ 1, pp. 287-296. 2016.

[25] S. Camarero, M.J. Martínez and A.T. Martínez. "Understanding lignin biodegradation for the improved utilization of plant biomass in modern biorefineries". Biofuels, Bioprod. Bioref. Vol. $8 \mathrm{~N}^{\circ} 5$, pp. 615-625. 2014. 
[26] A. Mariko, E. Takashi and H. Takahiro. "Crystalline transformation of native cellulose from cellulose I to cellulose II polymorph by a ball-milling method with a specific amount of water". Cellulose. Vol. $11 \mathrm{~N}^{\circ} 2$, pp. 162-7. 2004.

[27] E. Takács, L. Wojnárovits, C. Földváry, P. Hargittai, J. Borsa and I. Sajo. "Effect of combined gamma-irradiation and alkali treatment on cotton-cellulose". Radiat. Phys. Chem. Vol. $57 \mathrm{~N}^{\mathrm{o}}$ 3-6, pp. 399403. 2000.

[28] W. Seung, C. Eun, L. Dae-Seok, L. Soo, L. Young and B. Hyeun. "Lignocellulose conversion for biofuel: a new pretreatment greatly improves downstream biocatalytic hydrolysis of various lignocellulosic materials". Biotechnol. Biofuels. Vol. 8 $\mathrm{N}^{\circ} 228$, pp. 3-11. 2015.

[29] J. Leif and M. Carlos. "Pretreatment of lignocellulose: Formation of inhibitory byproducts and strategies for minimizing their effects". Bioresource Technology. Vol. 199, pp. 103-112. 2016.

[30] S. Muhammad, L. Jianghao, Z. Jiafu, H. Yan, W. Wen, C. Chang and L. Guangqing. "Improve the Anaerobic Biodegradability by Copretreatment of Thermal Alkali and Steam Explosion of Lignocellulosic Waste". BioMed Research International. Vol. 2016. Article ID 2786598. 10 pages. 2016. DOI: 10.1155/2016/2786598. 